\title{
Adaptación de un "Listado de verificación" para la realización de procedimientos en las Unidades del Dolor
}

\author{
J.M. Trinidad Martín-Arroyo ${ }^{1}$, A.I. Carnota Martín ${ }^{1}$, E. Calderón Seoane ${ }^{1}$, D. Benítez Pareja ${ }^{1}$, F. Aragón \\ Poce $^{1}$, J. Martínez Vázquez de Castro ${ }^{1}$ y L.M. Torres ${ }^{1,2}$ \\ ${ }^{1}$ Unidad del Dolor. Servicio de Anestesia y Reanimación. Hospital Universitario Puerta del Mar. Cádiz. \\ ${ }^{2}$ Departamento de Cirugía. Facultad de Medicina. Universidad de Cádiz. Cádiz
}

Trinidad Martín-Arroyo JM, Carnota Martín AI, Calderón Seoane E, Benítez Pareja D, Aragón Poce F, Martínez Vázquez de Castro J y Torres Morera LM. Adaptación de un "Listado de verificación" para la realización de procedimientos en las Unidades del Dolor. Rev Soc Esp Dolor 2015; 22(6): 275-280.

\section{INTRODUCCIÓN}

Desde que en 1999 el Instituto de Medicina reportara su artículo "To error is Human" (1), aparecieron alarmas y puntos de interés sobre la incidencia de errores médicos que mostraban la mortalidad y morbilidad relacionados con estos en EE. UU., y se puso en macha una serie de iniciativas para disminuir los mismos.

En 2003, the Joint Commision (2) propone un Protocolo Universal que los equipos quirúrgicos deben utilizar en cualquier cirugía electiva, y es en octubre de 2004 cuando la OMS (Organización Mundial de la Salud) crea una iniciativa especial conocida como "World Alliance for Patient Safety" con la intención de establecer políticas necesarias para mejorar la seguridad de los pacientes. Se encaró bajo el lema "La cirugía segura salva vidas" (Safe surgery save lives), un programa mundial para disminuir errores en la cirugía, y entre ellas, destaca la implementación de un listado de verificación quirúrgica (surgical safety checklist). El uso de dicho sistema ha sido evaluado posteriormente demostrando su efectividad y validez.

La iniciativa de la OMS pretende abordar cuestiones de seguridad importantes, como:
- Las prácticas inadecuadas de seguridad de la anestesia,

- Las infecciones quirúrgicas evitables o

- La comunicación deficiente entre los miembros del equipo quirúrgico.

Los conceptos fundamentales de esta iniciativa son: reconocimiento de la seguridad de la cirugía como un problema de salud, promover un enfoque sistémico de la seguridad de la cirugía y definir un conjunto básico de normas de seguridad que puedan aplicarse en todos los estados miembros de la OMS.

Para su desarrollo, se definieron cuatro áreas de trabajo en materia de: prevención de infecciones de la herida quirúrgica, seguridad de la anestesia, seguridad de los grupos quirúrgicos y medición de los servicios quirúrgicos. Posteriormente, se publicaron 10 objetivos básicos a cumplir en cualquier procedimiento quirúrgico (3):

- El equipo quirúrgico verifica que va a opera al paciente correcto en el sitio anatómico correcto.

- El equipo utiliza métodos reconocidos para prevenir daños derivados de la administración de anestésicos, mientras protege al paciente del dolor.

- El equipo se preparará eficazmente para una posible situación de pérdida de control de vía aérea y/o función respiratoria que amenace la vida del paciente, y reconocerá estas situaciones.

- El equipo se preparará y reconocerá situaciones que impliquen una potencial pérdida considerable de sangre del paciente.

- El equipo conocerá y estará preparado para cualquier reacción alérgica a fármacos o material que conoce suponen un riesgo vital para el paciente.

- El equipo se mantendrá alerta para evitar el depósito accidental de instrumental quirúrgico o compresas en el paciente. 
- El equipo guardará e identificará con precisión todas las muestras quirúrgicas.

- Los miembros del equipo mantienen una adecuada comunicación e intercambian datos críticos para una conducta segura durante ala operación.

- Los hospitales y sistemas públicos de salud establecerán vigilancia sistemáticas de la capacidad quirúrgica, volumen y resultados en los mismos.

En el análisis realizado en 8 hospitales ( 7.688 pacientes incluidos) sobre la implementación del listado de verificación quirúrgica, publicado por Haynes y cols. (4), se ha encontrado que el uso del checklist ha permitido un descenso en las complicaciones perioperatorias del 11 al $7 \%$ en pacientes mayores de 16 años para cirugía no cardiaca, y una disminución cercana al $40 \%$ de la mortalidad (reducción de la tasa del1,5 al 0,8\%). El estudio no permite identificar exactamente los mecanismos que conducen a estos resultados, pero sí implica una serie de cambios en el sistema quirúrgico, tanto en el comportamiento individual de los profesionales como en su relación y comunicación como equipo. Otro metaanálisis realizado posteriormente, desprende una disminución de la mortalidad quirúrgica entre el 47 y el $62 \%$, y un descenso de la morbilidad del paciente en torno al $37 \%$, identificando el trabajo en equipo, mejora de la comunicación y cultura de seguridad en los quirófanos como el eje fundamental de este cambio $(5,6)$.

La revisión del Protocolo Universal publicado en 2010, indica que el checklist debe ser realizado previo a cualquier bloqueo regional o procedimiento invasivo, centrándose en identificar el correcto sitio anatómico de bloqueo para evitar procedimientos erróneos o en lugares no adecuados. Del mismo modo, cualquier procedimiento invasivo tiene riesgo de complicarse con infección, efectos adversos que pongan en peligro la vida del paciente (reacciones alérgicas, toxicidad por fármacos...), efectos de anticoagulación, depresión respiratoria... que deben ser controlados y comprobados previo a la realización de cualquier procedimiento (7). Por este motivo, distintas organizaciones han desarrollado guías clínicas y recomendaciones para elaborar y aplicar un checklist de seguridad ante estas actuaciones, incluyendo la ASRA, ASA y la FDA.

\section{¿QUÉ ES UN LISTADO DE VERIFICACIÓN?}

"Es una serie de ítems que buscan identificar, planificar, comparar y verificar un conjunto de elementos, que permita, de manera oral o escrita, suplir las limitaciones de la memoria a corto plazo humana" (8). Los primeros listados de verificación en Medicina se basaron en los checklist utilizados de forma común en la industria de la aviación. Su objetivo era crear una herramienta que permitiera mejorar la seguridad del paciente dentro de la práctica clínica habitual. Esto implica la coordinación de todo el equipo de trabajo en los controles de seguridad en fases específicas, con el único objetivo de minimizar los riesgos inherentes a situaciones consideradas de riesgo, como actos quirúrgicos o procedimientos invasivos.

Según los requisitos de Joint Commision, un listado de verificación debe cubrir los tres momentos fundamentales en cualquier procedimiento: un registro antes del inicio del procedimiento (identificación del paciente, riesgos del mismo, procedimiento y sitio anatómico), un "minuto cero" justo previo a la realización del procedimiento (confirmación del equipo médico y material correcto, profilaxis de infecciones y eventos críticos previstos), y un cierre del proceso antes de que el paciente abandone la sala (confirmación del procedimiento realizado, contaje material y etiquetado de muestras, conformidad del equipo médico responsable) (9).

Las distintas asociaciones profesionales aconsejan adaptar cada modelo de verificación quirúrgica a las condiciones y peculiaridades locales de cada equipo u hospital, siguiendo una serie de recomendaciones en su adecuación y basándose en un modelo reconocido y aceptado por estas organizaciones.

\section{DESARROLLO DE UN LISTADO DE VERIFICACIÓN PARA PROCEDIMIENTOS INVASIVOS}

Los principios de la guía para la elaboración de un checklist son (10):

- Reducir la posibilidad de daño quirúrgico grave y evitable.

- Que no añada riesgos adicionales a la seguridad del paciente.

- Bajo coste de implantación.

- Simplicidad.

- Amplia aplicabilidad.

- Mensurabilidad.

El proceso de adaptación de un listado de verificación debe basarse en 5 puntos fundamentales: contenido y forma, timing, ensayo y feedback, evaluación y comprobación posterior, y modificaciones locales (11).

- Contenido y forma: el checklist debe recoger los ítems imprescindibles que deben ser controlados según la finalidad del mismo. Debe ser posible llevar a cabo tanto verificación oral como escrita en la sala de técnicas y corregir en ese momento los elementos detectados incorrectos. El objetivo general del checklist es centrarse en los "killer ítems", los cuales muestran elementos más críticos y potencialmente peligrosos para el paciente, tanto si son olvidados de revisar como si no están presentes en la preparación de la técnica.

- Timing: la experiencia de la aviación muestra que los humanos cometen errores y que el checklist debe per- 
mitir identificar y corregir los mismos antes de que causen daños (12). Para ello, el equipo debe identificar 3 fases en la dinámica de trabajo e instaurar unos "puntos pausa" dentro de la práctica clínica habitual: a la entrada en la sala de técnicas, antes del inicio del procedimiento invasivo, $\mathrm{y}$ antes de que el paciente abandone la sala. Sólo es necesaria la confirmación verbal de los pasos por parte del responsable designado por el equipo, que permita verificar el correcto funcionamiento del proceso antes de pasar al siguiente escalón.

- Ensayo y feedback: la estrategia está basada en la secuencia "Plan-Do_Study_Act" (PDSA), modelo de calidad validado en la industria e ingeniería. Durante el ensayo del checklist, el equipo clínico debe definir acciones y aclarar el lenguaje utilizado, corrigiendo términos confusos o demasiados extensos, primando la simplicidad y brevedad en la ejecución del listado.

- Prueba real y evaluación posterior: el uso del listado permitirá mejorar la adaptación del equipo a su aplicación, de forma que llegue a integrarse en la dinámica habitual de trabajo. La evaluación posterior suele realizarse desde la observación y la medición del proceso, más que en el registro escrito del checklist. Es mejor evaluar los efectos y mejora en la seguridad y cuidados, que valorar su "cumplimentación" como un éxito del proceso.
- Modificaciones locales: los listados de verificación no deben ser rígidos o prospectivos. Deben permitir la adaptación a las costumbres locales y procedimientos, mientras se mantengan los objetivos fundamentales y la brevedad en su aplicación. Estas modificaciones deben realizarse con la supervisión y colaboración de distintas disciplinas, que pueden focalizar sus objetivos en distintos ítems que ayuden a completar este listado de seguridad.

Esta adaptación requiere una rápida puesta en marcha del listado y posterior feedback en cada centro, para verificar su correcta modificación.

Como guía general para la realización-modificación de un checklist adaptado a las condiciones locales de cada centro, Gawande and Booman se proponen un esquema básico que se recoge en la figura 1 (13).

La implantación del listado de verificación es un proceso no exento de dificultades, ya que generalmente cualquier cambio en la práctica tradicional de un equipo genera rechazo, incluso algunos sanitarios pueden sentir que este análisis de seguridad pone en cuestión su profesionalidad. Para favorecer su correcta aceptación, se proponen una serie de medidas como (14):

- Lograr el apoyo de los líderes de la organización.

- Pilotar antes de implantar: elegir profesionales/servicios convencidos (líderes) pero con capacidad crítica.

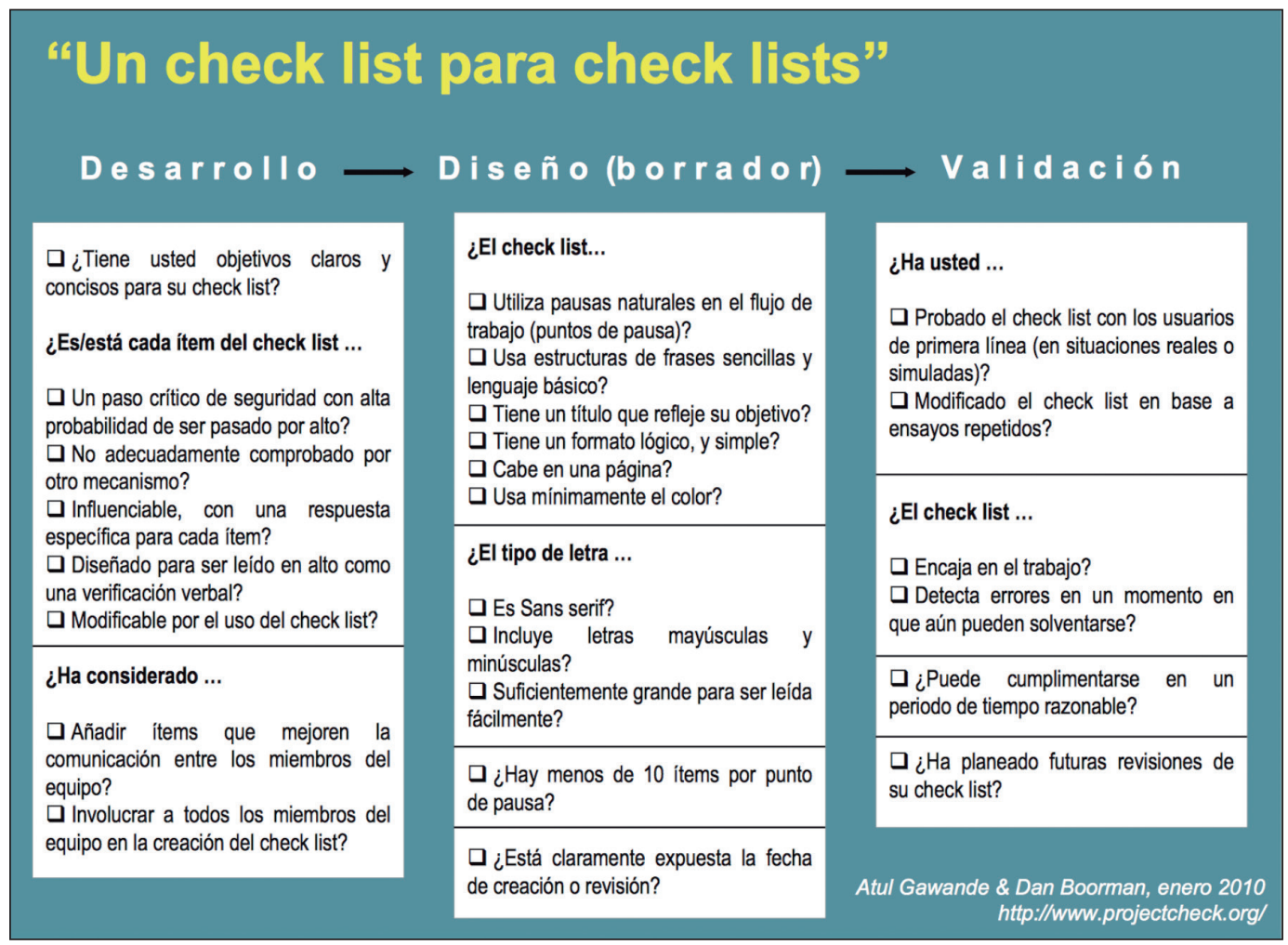

Fig. 1. "Checklist for Checklists" según www.projectcheck.org (13). 
- Llevar a cabo un plan formativo y de entrenamiento en el uso del checklist.

- Apoyar a los profesionales en el pilotaje, resolviendo dudas y designando un profesional responsable de su ejecución.

- Tras la implantación, revisar periódicamente su actualización y correcta aplicación.

El uso de listados de verificación en la práctica clínica sigue sumando importancia y se ha convertido en objeto de discusión y búsqueda de estrategias para desarrollar nuevos checklist en el campo de la medicina, de forma que se han realizado listados de seguridad en obstetricia, cuidados postquirúrgicos, cuidados intensivos y medicina de urgencia, como los listados para prevenir infecciones asociados a catéteres centrales o neumonía asociada a la ventilación mecánica $(15,16)$.

Sin embargo, la puesta en marcha de estos proyectos continúa sin ser fácil. Un reciente artículo que revisa los datos de 6.714 pacientes recogidos en 5 hospitales docentes, muestra como la adhesión al checklist quirúrgico continua siendo desigual e incompleta; comprobándose que al menos 1 de los 3 puntos del checklist quirúrgicos fueron rellenados (96,7\% casos), pero sólo se completo el $62,1 \%$ de los listados realizados. Se comprobó que estos datos no afectaban a la mortalidad, pero sí a la morbilidad del paciente, reduciéndola del $16,9 \%$ al $11,2 \%$. Se calcula que en torno al $14 \%$ de las complicaciones postoperatorias podrían haber sido prevenidas completando el listado de verificación correctamente (17).

Varios estudios muestran como la designación de un equipo multidisciplinar compuesto por enfermeras, cirujanos y anestesiólogos, mejora la implantación del listado de seguridad y el entrenamiento de todos los equipos en su cumplimentación, frente a los equipos de formación formados únicamente por un estamento, generalmente por enfermeras o auxiliares de quirófano $(18,19)$.

\section{PROPUESTA DE CHECKLIST EN BLOQUEOS REGIONALES DE LA ASRA}

Durante el Spring Meeting que organizó la Sociedad Americana de "Regional Anesthesia and Pain Medicine" (ASRA) en 2013, se recogieron la opinión de sus asistentes sobre la utilidad que tendría un checklist de seguridad previo a los bloqueos nerviosos. Para ello se recogió la opinión de los directores de programa de formación sobre anestesia regional y de los graduados en ese año, siendo esta última encuesta realizada a través de mail posterior al encuentro.

En el primer grupo, se obtuvo un $70 \%$ de participación entre los directores de programa, coincidiendo más de un $80 \%$ en la recomendación y utilidad que presenta un checklist de seguridad previo a los bloqueos regionales, y más de un $75 \%$ estuvieron de acuerdo/fuertemente de acuerdo con los ítems presentados, excepto en los referentes a la asepsia de la técnica, revisión del estado de no-resucitación y drogas necesarias para RCP previo a la realización del bloqueo. Respecto al grupo de los graduados, sólo un $33 \%$ participó en la encuesta solicitada a través de mail, y sus respuestas muestran que más del $75 \%$ se encuentra de acuerdo con la utilidad y necesidad de un listado de verificación previo a los bloqueos regionales, la necesidad de la correcta identificación del paciente, identificación y marcaje del lado correcto de la técnica y la necesidad de un repaso de seguridad antes de que el paciente abandone la sala de técnicas (7).

Se considera fundamental el primer ítem: "paciente correcto, lado correcto, apropiado marcaje", ya que estudios realizados en anestesia y dolor muestran una tasa del $0,02 \%$ de errores en este campo (209. Entre los factores que contribuyen a este error se encuentran el cambio de posición previo a la técnica, inadecuado marcaje del lugar de punción, cambios en el equipo que realizará la técnica, retraso o distracción entre la realización del checklist y la técnica, y lenguaje engorroso (21).

Otro punto fundamental se considera la revisión de la medicación habitual del paciente, así como la revisión de posibles alergias y enfermedades de interés del mismo. Se hace especial hincapié en revisar la historia clínica y corroborar la toma de anticoagulantes o HBPM, ya que estas prácticas siguen suponiendo un riesgo a pesar de las numerosas revisiones y guías clínicas realizadas por la ASRA y ASA. Debe revisarse y disponer de las principales drogas utilizadas en caso de RCP, y particularmente la disponibilidad de la emulsión lipídica para el tratamiento de una posible toxicidad por $\mathrm{AL}$, cuya ratio de presentación oscila en torno a 1:2000 técnicas locorregionales (22).

Así mismo, se recomienda seguir estrictamente las normas de asepsia básicas para cualquier técnica invasiva y realizar una "pausa" y revisión del checklist si va proceder con una segunda técnica, así como un repaso final antes de finalizar el procedimiento y dar de alta al paciente, siendo este un punto aún por conseguir dentro de la práctica anestésica.

\section{NUESTRA EXPERIENCIA EN LA UNIDAD DEL DOLOR}

En los últimos años, las Unidades del Dolor se han desarrollado extraordinariamente, $y$ al mismo tiempo ha supuesto un reto para la organización y gestión de las mismas. El aumento de la cartera de servicio con la incorporación de múltiples nuevos procedimientos intervencionistas, ecoguiados o radioguiados, ha obligado a un proceso de formación y especialización de nuestros profesionales pero, a la vez, ha supuesto un aumento de la demanda de nuestras Unidades; sin olvidar, la necesaria reestructuración de las 
instalaciones para adaptarlas a estas técnicas, manteniendo los estándares de seguridad y calidad.

Actualmente, la sala de técnicas/quirófano de las unidades del dolor, tienen una alta carga asistencial en la que durante una jornada se pueden llevar a cabo numerosos procedimientos. Así pues, este reto en innovación y demanda de nuestros servicios, nos obliga a no descuidar los aspectos básicos en seguridad del paciente, y entre ellos, una de las herramientas principales y que se ha demostrado sencilla y eficaz es el "listado de verificación" o "checklist".

De este modo, partiendo de la adaptación del "checklist" para bloqueos periféricos publicada en el Regional Anesthesia and Pain Medicine, decidimos implementar un modelo de "listado de verificación" específico para los procedimientos de tratamiento del dolor (bloqueos, radiofrecuencia, adhesiolisis, epiduroscopia,....) que realizábamos en nuestra unidad, siendo el mismo puesto en marcha en septiembre de 2014.

El listado de verificación consta de 3 fases:

- Una primera al recepcionar al paciente en la unidad y que realiza la enfermera de la sala de observación.

- Una segunda que se realiza en la sala de técnicas/quirófano, previo al inicio del procedimiento de forma conjunta entre enfermera, técnico de rayos y médico. No se entregan las agujas de radiofrecuencia/bloqueo hasta que se ha verificado su cumplimentación de forma oral y conjunta.

- Una tercera en la sala, al finalizar el procedimiento.

Desde entonces, se ha usado el "listado de verificación adaptado a Unidad del Dolor" en 192 pacientes a los que se han realizado técnicas invasivas para tratamiento del dolor crónico en nuestra unidad. Durante este tiempo, se han evitado 3 casos de "lado erróneo" en la segunda fase/pausa del listado de verificación. Con ello, no se ha registrado ningún caso de equivocación del lado tratado o problemas en la identificación del paciente. Además ha permitido detectar tratamientos anticoagulantes no registrados con anterioridad y por tanto, hizo que se postpusiera el procedimiento hasta la semana siguiente en la que se cumplieran las condiciones de seguridad. También ha mejorado las condiciones de monitorización y control del paciente, permitiendo un abordaje más ágil y seguro de los eventuales síndromes vagales acaecidos u otras incidencias.

Dicho listado ha sido validado por la Comisión de Seguridad del Hospital Universitario Puerta del Mar e incorporado a la documentación de la web corporativa (Fig. 2).

\section{CONCLUSIÓN}

La seguridad del paciente se ha convertido en una prioridad en la gestión de nuestras unidades. Los procedimientos que se realizan en las unidades del dolor se han multiplica-

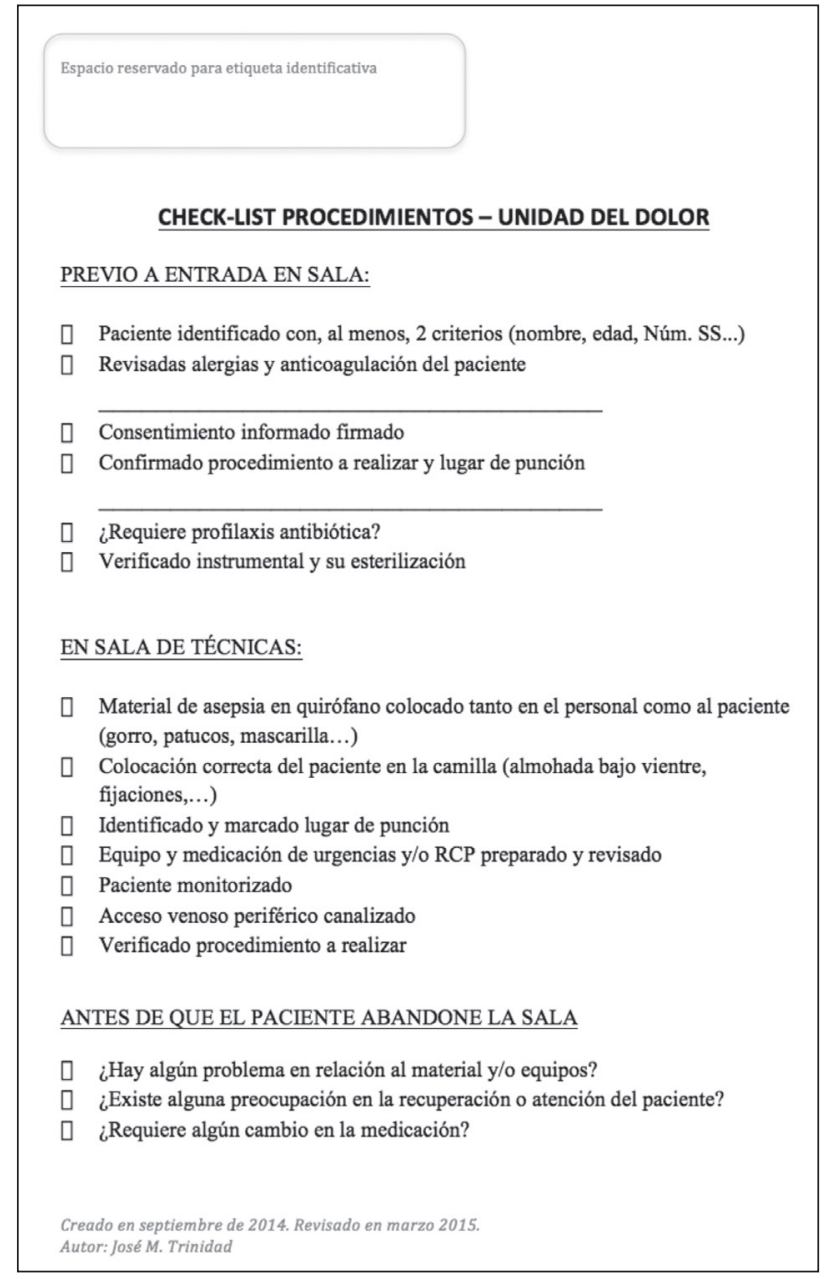

Fig. 2. Propuesta de listado de verificación específico para Unidades del Dolor. Hospital Univ. Puerta del Mar (Cádiz).

do en los últimos años, por lo que para evitar la incidencia de complicaciones hemos de desarrollar métodos que ya se vienen usando desde hace tiempo en otros medios con alto grado de eficacia. Entre estos, consideramos imprescindible el uso sistematizado de estos listados de verificación.

Nosotros hemos desarrollado un "checklist" específico y adaptado a nuestro medio, en el que tenemos la sala de técnicas radio/ecoguiadas integrada en la propia unidad del dolor y nuestro equipo de enfermería es quien gestiona la recepción y cuidado post-procedimiento de los pacientes. Por ello, cada centro ha de adaptar dicho listado de verificación a las características de su propia unidad.

Las limitaciones en la implementación de un "listado de verificación" son ya conocidas y se ha hablado de ellas. No obstante, habrá que trabajar con el equipo hasta que se convierta en una actividad rutinaria. Existen herramientas que pueden ayudar a su implementación, como por ejemplo, que la enfermera no facilite las agujas de bloqueo/ radiofrecuencia hasta que se haya realizado la pausa y cumplimentado los items del listado previo. 
Como conclusión, consideramos imprescindible sistematizar el uso de un "checklist" específico y adaptado a nuestro medio en las Unidades del Dolor y evaluar posteriores revisiones del mismo, para lograr estándares óptimos de seguridad del paciente en nuestra especialidad.

\section{AGRADECIMIENTOS}

A todo el equipo de enfermería y auxiliar de enfermería de la Unidad del Dolor del Hospital Universitario Puerta del Mar, que con su compromiso diario, hacen posible la implementación de nuevas herramientas de mejora.

\section{CORRESPONDENCIA:}

José Manuel Trinidad Martín-Arroyo

Unidad del Dolor.

Servicio de Anestesiología y Reanimación.

Hospital Universitario Puerta del Mar

Avda. Ana de Viya, 21

11010 Cádiz

e-mail: jmtrinidad80@gmail.com

\section{BIBLIOGRAFÍA}

1. Kohn LT, Corrigan JM, Donaldson MS, editors. To Err Is Human: Building a Safer Health System. Washington, DC: National Academy Press; 2000.

2. Universal Protocol. Available at: http://www.jointcommission.org/ standards_information/up.aspx, Accessed June 6, 2013.

3. Schlack WS, Boermeester MA. Patient safety during anaesthesia: Incorporation of the WHO safe surgery guidelines into clinical practice. Curr Opin Anesthesiol 2010;23:754-8.

4. Haynes AB, et al. A surgical safety checklist to reduce morbidity and mortality in a global population. N Engl J Med 2009;360:491-9.

5. Fudickar A, et al. The effect of the WHO Surgical Safety Checklist on complication rate and communication. Dtsch Arztebl Int 2012;109(42):695-701.

6. Treadwell JR, Lucas S, Tsou AY. Surgical checklists: A systematic review of impacts and implementation. BMJ Qual Saf 2014;23:299-318.
7. Mulroy MF, et al. A checklist for preforming regional nerve blocks. Reg Anesth Pain Med 2014;39:195-9.

8. Federal Aviation Administration. Section 12: Aircraft Checklist for 14 CFR Parts 121/135. iFOFSIMSF.

9. Manual de aplicación de la lista OMS de verificación de la seguridad de la cirugía 2009: la cirugía segura salva vidas. Organización Mundial de la Salud. ISBN 978-9243598598.

10. Guía para la incorporación de la seguridad clínica a aquellos pacientes que van a ser intervenidos quirúrgicamente en el SSPA. Comité operativo para la seguridad de pacientes de Andalucía. Consejería de Salud de la Junta de Andalucía. Diciembre 2009.

11. Weiser TG, et al. Perspectives in quality: designing the WHO Surgical Safety Checklist. International Journal for Quality in Health Care 2010;22(5):365-70.

12. Gawande A. The Checklist Manifesto: How to Get Things Right. Metropolitan Books, New York: Holt and Company; 2009.

13. www.projectcheck.org. Atul Gawande \& Dan Booman, January 2010.

14. Hales BM, et al. Development of medical checklists for improved quality of patient care. Int J Qual Health Care 2008;20(1):22-30.

15. Hales BM, Pronovost PJ. The checklist - a tool for error management and performance improvement. J Crit Care 2006;21: 231-5.

16. Winters BD, Gurses AP, Lehmann H, et al. Clinical review checklists - translating evidence into practice. Crit Care 2009; $13: 210$.

17. Mayer EK, et al. Surgical Checklist Implementation Project: The Impact of Variable WHO Checklist Compliance on Risk-adjusted Clinical Outcomes After National Implementation: A Longitudinal Study. Ann Surg 2015 Mar 13.

18. Hannam JA, et al. A prospective, observational study of the effects of implementation strategy on compliance with a surgical safety checklist. BMJ Qual Saf 2013;22(11):940-7.

19. Mahajan RP. The WHO surgical checklist. Best Pract Res Clin Anaesthesiol 2011;25(2):161-8.

20. Cohen SP, et al. Incidence and root cause analysis of wrongsite pain management procedures: A multicenter study. Anesthesiology 2010;112:711-8.

21. Russ SJ, et al. A qualitative evaluation of the barriers and facilitators toward implementation of the WHO Surgical Safety Checklist across hospitals in England. Lessons from the "Surgical Checklist Implementation Project". Ann Surg 2014;00:1-11.

22. Sites BD, et al. Incidence of local anesthesic systemic toxicity and postoperative neurologic symptoms associated with 12,668 ultrasound-guided nerve block: An analysis from prospective clionical regristry. Reg Anesth Pain Med 2012;37:478-29. 